\title{
Vorbild Silicon Valley
}

\section{Liebe Leserin, lieber Leser,}

Silicon Valley, die Region in Kalifornien, ist zum Synonym für Innovationen und unternehmerische Leitkultur geworden - mittlerweile auch über die namensgebenden Computer-, Elektronik- und Halbleitertechniken hinaus. Ein AutoMobil-Entwicklungsstandort der neuen Art. Andere IT-Cluster verwenden marketing- oder auch mediengetrieben „Silicon“ oder „Valley“, um eine Verbindung zum Original herzustellen, beispielsweise Silicon Alps - Kärnten, Isar Valley - München oder Indisches Silicon Valley - Bangalore.

Der IT-Cluster Rhein-Main-Neckar, die Region zwischen Frankfurt am Main, Darmstadt, Mannheim, Karlsruhe und Heidelberg wird nachvollziehbar als „Silicon Valley Europas“ bezeichnet, da sich in der Region der größte SoftwareCluster Europas gebildet hat. Der Universitätsstandort Bochum gilt unter Insidern als das Silicon Valley für Security-Nachwuchskräfte, die wiederum vom originalen Silicon Valley rekrutiert werden ein deutscher Exportschlager.

Das Original zieht dennoch immer mehr deutsche Unternehmen an, nicht nur große Firmen wie Daimler, die Deutsche Telekom oder Siemens. Auch klein- und mittelständische Unternehmen, Institute und zahlreiche Berater gründen Büros in der kalifornischen Ebene, mehr als in anderen US-Bundesstaaten, um unter anderem nah an künftigen Kunden, Start-ups und denen, die es werden können, zu sein.

Doch die Menge und Vielfalt an potenziellen Partnerschaften allein scheinen nicht den Ausschlag zu geben. Hochrangige deutsche Entscheidungsträger berichten beispielsweise von einem in Deutschland unerreichten Spirit, einem begeisterungsfähigen Pragmatismus und einem offenen Netzwerken, für das eigentlich die deutsche Industrie inter- national anerkannt wird. Sie zitierten den oft erwähnten Mut, ein Scheitern zu riskieren, der auf dem Weg zum Erfolg irgendwie in Kauf genommen wird. Ganz entgegen der Nullfehlerkultur, die Ex-Volkswagen-Chef Martin Winterkorn fatalerweise auf die Spitze trieb.

Einige der Entscheidungsträger haben an der Universität Stanford studiert. Einer von ihnen saß im Aufsichtsrat eines Start-ups. Die Frage, was er vom Silicon-Valley-Unternehmen in die Fahrzeugentwicklung im deutschen Zuhause mitnehmen und dort umsetzen kann, offenbarte eine gewisse Ratlosigkeit. Ein Transfer sei denkbar unmöglich, zu verschieden funktionierten die Systeme und Unternehmenskulturen. Ich glaube hingegen, in der Offenheit, Flexibilität und Integrationsfähigkeit liegt die Kunst künftiger Erfolge.

Diese Meinung werden viele nicht teilen. Sie mögen auf langjährige intensive „interkulturelle“ Erfahrungen verweisen. Eine Diskussion kann deshalb spannend sein - allerdings vor dem Hintergrund, dass sich das „System Auto“ laut Branchenexperten neu erfinden muss.

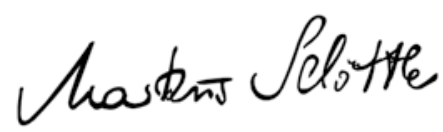

Markus Schöttle

Stellvertretender Chefredakteur

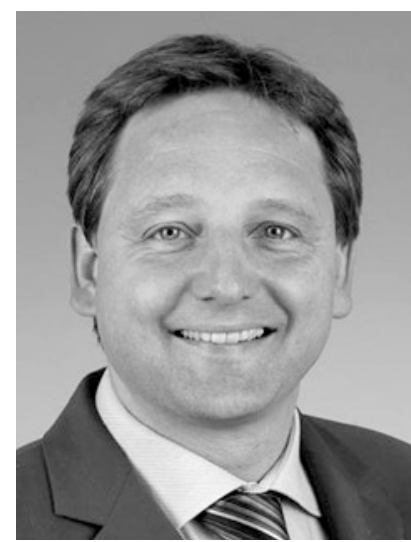

Tonangebend

\section{in der optischen}

Schwingungsmessung

\section{Noch verlässlicher, noch vielseitiger, noch besser}

